\title{
CVD growth of sp2-hybridized boron nitride using aluminum nitride as buffer layer
}

\author{
Mihails Cubarovs, Henrik Pedersen, Hans Högberg, Vanya Darakchieva, \\ Jensen Jens, Per Persson and Anne Henry
}

\section{Linköping University Post Print}

N.B.: When citing this work, cite the original article.

Original Publication:

Mihails Cubarovs, Henrik Pedersen, Hans Högberg, Vanya Darakchieva, Jensen Jens, Per Persson and Anne Henry, CVD growth of sp2-hybridized boron nitride using aluminum nitride as buffer layer, 2011, Physica Status Solidi. Rapid Research Letters, (5), 10-11, 397 399.

http://dx.doi.org/10.1002/pssr.201105410

Copyright: Wiley-VCH Verlag Berlin http://www.wiley-vch.de/publish/en/

Postprint available at: Linköping University Electronic Press http://urn.kb.se/resolve?urn=urn:nbn:se:liu:diva-73160 
2

\title{
Epitaxial CVD growth of $\mathrm{sp}^{2}$ - hybridized boron nitride using aluminum nitride as buffer layer
}

\author{
Mikhail Chubarov ${ }^{\star}$, Henrik Pedersen, Hans Högberg, Vanya Darakchieva, Jens Jensen, \\ Per O. Å. Persson, Anne Henry
}

Department of Physics, Chemistry and Biology, Linköping University, SE-581 83 Linköping, Sweden

Received ZZZ, revised ZZZ, accepted ZZZ

Published online ZZZ (Dates will be provided by the publisher.)

Keywords CVD, h-BN, r-BN, Epitaxy

* Corresponding author: e-mail: mihails.cubarovs@liu.se, Phone: +46 1328 8983, Fax: +4613137568

\begin{abstract}
Epitaxial growth of $\mathrm{sp}^{2}$-hybridized boron nitride (BN) using chemical vapour deposition, with ammonia and triethyl boron as precursors, is enabled on sapphire by introducing an aluminium nitride (AlN) buffer layer. This buffer layer is formed by initial nitridation of the substrate. Epitaxial growth is verified by $\mathrm{X}$-Ray diffraction measurements in Bragg - Brentano configuration, pole
\end{abstract}

figure measurements and transmission electron microscopy. The in plane stretching vibration of $\mathrm{sp}^{2}$-hybridized BN is observed at $1366 \mathrm{~cm}^{-1}$ from Raman spectroscopy. Time of flight elastic recoil detection analysis confirms almost perfect stoichiometric BN with low concentration of carbon, oxygen and hydrogen contaminations.

Copyright line will be provided by the publisher

1 Introduction Boron nitride $(\mathrm{BN})$ is a multifunctional material consisting of equal proportion of $\mathrm{B}$ and $\mathrm{N}$ atoms. BN has a number of excellent properties analogue to diamond and graphite such as mechanical properties but also wide band gap [1;2], low dielectric constant, piezoelectricity and good stability at high temperatures [3]. Further, BN can easily be doped p- and n-type for electronic applications [2]. It is thus a promising material for many applications. However, BN is one of the least investigated semiconductors among the group III-nitrides. In the same way as carbon exists as graphite and diamond, BN can occur in $\mathrm{sp}^{2}$-bonded and $\mathrm{sp}^{3}$-bonded structures. The $\mathrm{sp}^{3}$ bonded forms include cubic (c-BN) and wurzitic (w-BN) phases whereas the hexagonal (h-BN) and the rhombohedral (r-BN) are the $\mathrm{sp}^{2}$-bonded phases. The h-BN form has a layer structure with a two-layered stacking sequence ( $\mathrm{ABAB} \ldots$ structure) whereas the $\mathrm{r}-\mathrm{BN}$ phase has a threelayered stacking sequence (ABCABC... stacking). The fact that $\mathrm{h}-\mathrm{BN}$ and $\mathrm{r}-\mathrm{BN}$ have very similar spacing between the basal planes ( $3.325 \AA$ and $3.33 \AA$, respectively) [4] and equal in-plane lattice parameter of $2.50 \AA$, makes distinction between them extremely difficult. Thus, they cannot be distinguished by common techniques as X-Ray diffraction (XRD) in Bragg - Brentano configuration, transmis- sion electron microscopy (TEM), Raman spectroscopy, Fourier transform infrared spectroscopy (FTIR) or any composition measurements.

Films with $\mathrm{sp}^{3}$-bonded form (mainly c-BN) are generally deposited with "brutal force" such as by energetic ionassisted physical vapour deposition. The use of more gentle deposition processes will favour the formation of $\mathrm{sp}^{2}$ hybridized structures. Synthesis of highly crystalline r-BN nanoplates has been reported [5], but monocrystallie epilayers remains to be demonstrated, to the best of our knowledge. Kobayashi et al. [2;6;7] showed that h-BN can be epitaxially grown by metal-organic chemical vapor deposition (MOCVD) on sapphire $\left(\alpha-\mathrm{Al}_{2} \mathrm{O}_{3}\right)(0001), \mathrm{Ni}$ (111) and 6H-SiC (0001) using triethyl boron (TEB) $\left(\mathrm{B}\left(\mathrm{CH}_{2} \mathrm{CH}_{3}\right)_{3}\right)$ and ammonia $\left(\mathrm{NH}_{3}\right)$ as precursors. However, their presented XRD data showed very broad h-BN peak. Younes et al. [8] presented CVD growth of h-BN on $6 \mathrm{H}-$ $\mathrm{SiC}$ and $4 \mathrm{H}-\mathrm{SiC}$ on-axis (0001) substrates using diborane $\left(\mathrm{B}_{2} \mathrm{H}_{6}\right)$ and ammonia with $\mathrm{Ar}$ as carrier gas. Broad h-BN related peaks with very low intensity characterized the XRD patterns and their TEM investigations revealed nanocrystalline rather than epitaxial growth of the film. Rye demonstrated hot filament activated CVD of polycrystalline h-BN from borazine $\left(\mathrm{B}_{3} \mathrm{~N}_{3} \mathrm{H}_{6}\right)$ [9], however, no XRD 
measurements were presented although Raman spectroscopy showed a peak from $\mathrm{sp}^{2}$-hybridized $\mathrm{BN}\left(\mathrm{sp}^{2} \mathrm{BN}\right)$. Recently, R. Dahal et al. [10] presented data on the epitaxial growth of h-BN, but confirmation of epitaxy is done by only XRD measurements in Bragg - Brentano configuration.

For the growth of $\mathrm{sp}^{2} \mathrm{BN}$ as semiconductor device material, the substrate should have a low lattice mismatch to $\mathrm{h}-\mathrm{BN}$ and $\mathrm{r}-\mathrm{BN}$ and be applicable to the electronic device manufacturing. Wafers of $\alpha-\mathrm{Al}_{2} \mathrm{O}_{3}$ and $6 \mathrm{H}-\mathrm{SiC}$ are both used in devices based on III-N materials today, sapphire being the less expensive choice. Both types of substrates have a significant lattice mismatch to $\mathrm{sp}^{2} \mathrm{BN}$ (lattice constants of $4.67 \AA$ and $3.10 \AA$, respectively [4]). Single crystal $\mathrm{Ni}$ (111) shows a better lattice match to $\mathrm{sp}^{2} \mathrm{BN}(0001)$ (lattice constants of $2.49 \AA$ and $2.50 \AA$, respectively [4,6]).

In this work we apply an AlN buffer layer on sapphire from this the lattice mismatch to $\mathrm{sp}^{2} \mathrm{BN}$ is reduced and the growth of epitaxial $\mathrm{sp}^{2} \mathrm{BN}$ crystalline films is enabled.

2 Experimental A hot wall chemical vapour deposition (CVD) reactor with a $\mathrm{SiC}$ coated graphite susceptor was used for the growth. The pressure during deposition was 100 mbar and $\mathrm{H}_{2}$ was used as carrier gas. It was noted that the reactive chemical environment supplied by the hydrogen gas was needed, since no $\mathrm{BN}$ film was formed in experiments with an inert atmosphere of nitrogen or argon as carrier gas. Ammonia and TEB were used as precursor gases. Typical $\mathrm{B} / \mathrm{H}_{2}$ molar fraction was $0.02 \%$ and a N/Bratio of 600 was used. Sapphire substrates, $\alpha-\mathrm{Al}_{2} \mathrm{O}_{3}(0001)$, were employed at a typical growth temperature of $1500{ }^{\circ} \mathrm{C}$. In order to reduce the lattice mismatch between $\alpha-\mathrm{Al}_{2} \mathrm{O}_{3}$ and $\mathrm{sp}^{2} \mathrm{BN}$ (in-plane axis $4.67 \AA$ and $2.50 \AA$ respectively [4]) an AlN (in-plane axis $3.11 \AA$ [4]) buffer layer was introduced. Formation of the AlN layer was achieved through nitridation of the $\alpha-\mathrm{Al}_{2} \mathrm{O}_{3}$ surface $[11 ; 12]$ by introducing ammonia in the reactor with a $\mathrm{NH}_{3} / \mathrm{H}_{2}$ molar fraction of $0.13 \%$ at the growth temperature for 10 minutes. The deposition of the BN film was initiated by adding TEB to the gas mixture and adjusting the ammonia flow after the nitridation step to obtain the required N/B ratio.

For characterization of the grown films, XRD in Bragg - Brentano configuration $(\theta-2 \theta)$, pole figure measurements, TEM with energy dispersive X-ray spectroscopy (EDX), Raman spectroscopy with $363.84 \mathrm{~nm}$ excitation laser and time of flight elastic recoil detection analysis (ToFERDA) were applied.

3 Results and discussion Figure 1 presents diffractograms of the films grown on non nitridized sapphire substrate (FIG 1. A) and on nitridized sapphire (FIG 1. B), respectively. Only a peak from turbostratic $\mathrm{BN}(\mathrm{t}-\mathrm{BN})$ at $26.3^{\circ}$ is observed in the case of non nitridized sapphire substrate (FIG 1. A) while the film grown on nitridized substrate exhibited (0001) orientated $\mathrm{sp}^{2} \mathrm{BN}$ as evident from the XRD pattern (FIG. 1 B). Two peaks are thus observed which are related to either h-BN or $\mathrm{r}-\mathrm{BN}$ at $26.7^{\circ}$

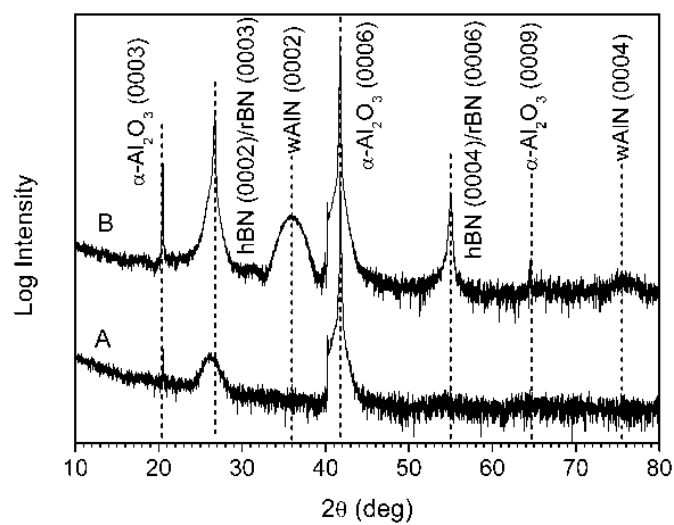

Figure $1 \mathrm{X}$-Ray diffractogram in Bragg - Brentano configuration of the $\mathrm{sp}^{2} \mathrm{BN}$ film grown on sapphire without AlN buffer layer (A) and with AlN buffer layer (B).

(0002 of h-BN or 0003 of r-BN) and $55.1^{\circ}$ (0004 of h-BN or 0006 of r-BN), respectively [4]. The calculated spacing between the basal planes from the XRD-data is $3.30 \pm 0.05$ $\AA$. The XRD result suggests that the grown $\mathrm{sp}^{2} \mathrm{BN}$ film is highly oriented since only peaks from the (0001) planes are observed for this measurement. Also for the AlN buffer layer only peaks originated from the (0002) and (0004) planes are found at $36.0^{\circ}$ and $76.2^{\circ}$ respectively, indicating that the AlN formed by nitridation grows as AlN (0001) on $\alpha-\mathrm{Al}_{2} \mathrm{O}_{3}(0001)$, as previously reported [11;12].

As seen in FIG. 1, the AlN peaks are significantly broadened, which is an indication of a thin film as observed in the TEM image (FIG.2). A slight asymmetry on the left side of the peak at $26.72^{\circ}$ of the $\mathrm{sp}^{2} \mathrm{BN}$ is most likely due to the minor inclusions of less ordered material, t-BN, near to the interface with AlN in order to compensate the lattice mismatch. The asymmetry in this case can also be explained by point defects in $\mathrm{sp}^{2} \mathrm{BN}$ near to the interface with AlN.

The cross sectional TEM image (FIG. 2) confirmed the epitaxial growth of $\mathrm{sp}^{2} \mathrm{BN}$ on the AlN buffer layer. In the micrograph the AlN and $\mathrm{sp}^{2} \mathrm{BN}$ planes are clearly discernible and allow a rough estimate of the c-axis lattice parameters for $\mathrm{AlN}$ and plane spacing for $\mathrm{sp}^{2} \mathrm{BN}$ which were found to be $2.45 \pm 0.05 \AA$ and $3.36 \pm 0.05 \AA$, respectively.

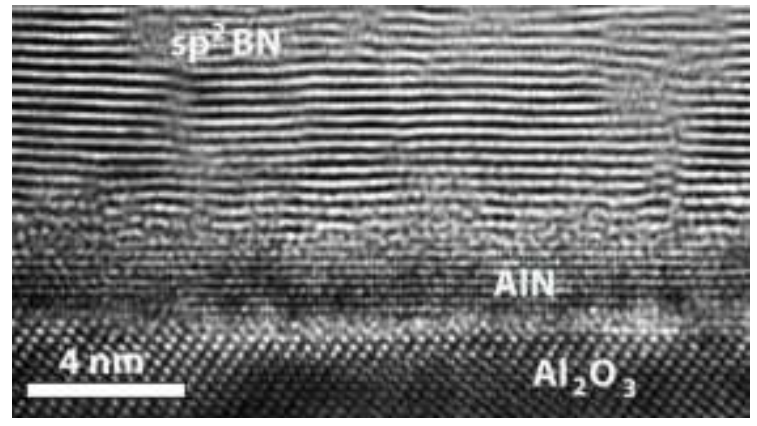

Figure 2 Cross section TEM micrograph of the $\mathrm{Al}_{2} \mathrm{O}_{3} / \mathrm{AlN} / \mathrm{BN}$ interface region. 


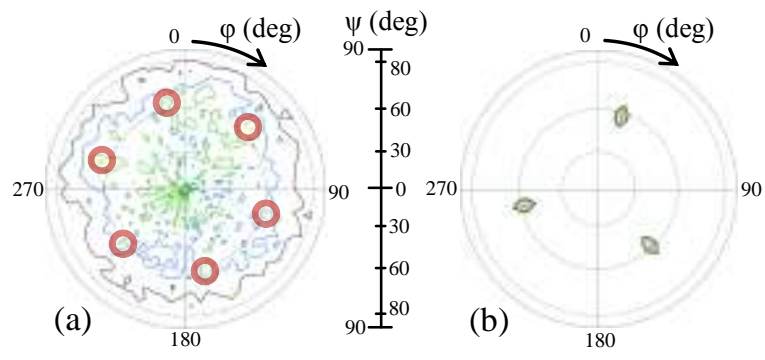

Figure 3 Pole figures of $\{01 \underline{12}\}$ planes of (a) r-BN and (b) Sapphire. The red circles are for a better visualization of the peak positions.

These lattice parameters for $\mathrm{AlN}$ and $\mathrm{sp}^{2} \mathrm{BN}$ are close to the previously reported $2.49 \AA$ and $\sim 3.33 \AA$, respectively [4]. It can also be noted that the c-axis parameter in AlN deviates more from the reference value close to the sapphire. Composition measurements using EDX showed a significant concentration of oxygen in the AlN buffer layer and in the interface between $\mathrm{BN}$ and AlN. The interface between BN and AlN appears less ordered in the micrograph. This may be attributed to initially different nucleation orientations or surface roughness, which is soon replaced by ordered epitaxial growth. Some grain boundaries can be seen in the $\mathrm{sp}^{2} \mathrm{BN}$ film, giving rise to seemingly less ordered features.

Pole figure measurements of $\{0112\}$ planes of h-BN and $\mathrm{r}-\mathrm{BN}$ revealed that the film contains mainly $\mathrm{r}-\mathrm{BN}$. It is worth to note, that the detection of h-BN asymmetric reflections in the pole figure measurements is a complicated task since the week intensity peaks of h-BN overlap with long tails of the high intensity sapphire asymmetric peaks. It is well known fact that AlN epitaxialy grown on sapphire is $30^{\circ}$ rotated around the c-axis with respect to the sapphire lattice and it is also true for nitridized sapphire [13]. From the pole figure measurements done on $\mathrm{sp}^{2} \mathrm{BN}$ samples and the fact that AlN crystal lattice is $30^{\circ}$ rotated with respect to the sapphire lattice, it can be concluded that r-BN crystal directly grows on AlN layer and the r-BN crystal is thus $30^{\circ}$ rotated with respect to sapphire (FIG. 3). Raman scattering measurements showed $\mathrm{sp}^{2}$-hybridized $\mathrm{B}$ $\mathrm{N}$ stretching vibrations within the basal planes at $1366 \mathrm{~cm}^{-1}$ and a full width at half maximum of $31 \mathrm{~cm}^{-1}$. This Raman peak position is identical to the previously reported data for h-BN powder [14], however a similar peak position is also expected for r-BN material. Broadening of the Raman peak is probably due to the twin structure of the $\mathrm{r}-\mathrm{BN}$ and also due to the inclusions of t-BN which are distortions in the periodicity of the crystal. Evidence of the twin structure of the r-BN crystal is observed by pole figure measurements (FIG.3 a), since only 3 reflections should be observed for r-BN $\{01 \underline{12}\}$ planes. This phenomenon has been previously observed for CVD grown r-BN nanoparticles [15].
The thickness of the film, as evaluated from TEM images is about $400 \mathrm{~nm}$, corresponding to a growth rate of $100 \mathrm{~nm} / \mathrm{h}$.

The elemental composition in the bulk of the film, as evaluated by ToF-ERDA, was found to be 50 at $\%$ B and 49 at $\%$ N. This is, within the experimental uncertainty of the measurement, very close to what is expected for BN (uncertainty is $1-1.5$ at $\%$ for these elements). The main observed impurities were $\mathrm{H}, \mathrm{O}(\sim 0.1$ at $\%)$ and $\mathrm{C}(<0.1$ at $\%$ close to the detection limit), in total about 1 at $\%$ with $\mathrm{H}$ being the main contamination element.

4 Conclusions In summary, r- or h-BN with high crystalline quality was grown epitaxially by CVD on $\alpha$ $\mathrm{Al}_{2} \mathrm{O}_{3}$ (0001) substrates. The epitaxial growth was enabled by introducing an AIN buffer layer which is formed through the transformation of the sapphire surface by nitridation. These results announce the possibility to further explore $\mathrm{sp}^{2} \mathrm{BN}$ for use in electronic applications.

Acknowledgements Dr. Ivan G. Ivanov is acknowledged for assistance with Raman measurements. Prof. Karin Larsson and Prof. Erik Janzén are acknowledged for helpful discussions. The Swedish Research Council (VR 621-2009-5264) is gratefully acknowledged for financial support.

\section{References}

0 [1] M. G. Silly, P. Jaffrennou, J. Barjon, J.-S. Lauret, F. Ducastelle, A. Loiseau, E. Obraztsova, B. Attal-Tretout, and E. Rosencher, Phys. Rev. B 75, 085205 (2007)

[2] Y. Kobayashi, T. Nakamura, T. Akasaka, T. Makimoto, N. Matsumoto, J. Crystal Growth 298, 325 - 327 (2007)

[3] S. Zhang, G. Chen, B. Wang, D. Zhang, H. Yan, J. Crystal Growth 223, 545-549 (2001)

[4] Joint Committee on Powder Diffraction Standards, JCPDS, Swarthmore, PA, pattern 34 - 0421, pattern 45 - 1171, pattern $83-2080$, pattern $75-1541$, pattern $25-1133$

[5] K. Bao, F. Yu, L. Shi, S. Liu, X. Hu, J. Cao, and Y. Qian, J Sol Stat Chem 182, 925-931 (2009)

[6] Y. Kobayashi T. Akasaka, J. Crystal Growth 310, 5044 5047 (2008)

[7] Y. Kobayashi, T. Akasaka, T. Makimoto, J. Crystal Growth 310, 5048 - 5052 (2008)

[8] G. Younes, G. Ferro, M. Soueidan, A. Brioude. F. Cauwet, Materials Science Forum 645 - 648 , 1191 - 1194 (2010)

[9] R. R. Rye, J. Vac. Sci. Technol. A 9 (3), 1099 - 1103 (1991)

[10] R. Dahal, J. Li, S. Majety, B. N. Pantha, X. K. Cao, J. Y. Lin, H. X. Jiang, Appl. Phys. Lett. 98, 211110 (2011)

[11] P. Vennéguès, B. Beaumont, Appl. Phys. Lett. 75 (26), 4115-4117 (1999)

[12] K. Akiyamaa, Y. Ishii , H. Murakami, Y. Kumagai, A. Koukitu, J. Crystal Growth 311, 3110-3113 (2009)

[13] H.Fukuyama, S. Kusunoki, A. Hakomori, K. Hiraga, J. Appl. Phys. 100, 024905 (2006)

[14] T. Kuzuba, K. Era, T. Ishii, T. Sato, Sol. Stat. Com. 25, 863 -865 (1978)

[15] T. Oku, K. Hiraga, T. Matsuda, T. Hirai, M. Hirabayashi, Diamond and Related Materials 12, 1138 - 1145 (2003) 